\title{
How one institution overcame the challenges to start an MRI-based brachytherapy program for cervical cancer
}

\author{
Matthew M. Harkenrider, MD!, Steven M. Shea, PhD², Abbie M. Wood, PhD', Bonnie Chinsky, MS', Amishi Bajaj, BA!', \\ Michael Mysz, MS', Joseph H. Yacoub, MD², Ari Goldberg, MD, PhD², Margaret Liotta, DO ${ }^{3}$, Ronald Potkul, MD, FACOG ${ }^{3}$. \\ Murat Surucu, PhD!, John Roeske, PhD!, William Small Jr., MD, FACRO, FACR, FASTROl \\ 'Department of Radiation Oncology, ${ }^{2}$ Department of Radiology, ${ }^{3}$ Department of Obstetrics and Gynecology, Stritch School of Medicine \\ Loyola University Chicago, Maywood, IL, USA
}

\begin{abstract}
Purpose: Adaptive magnetic resonance imaging (MRI)-based brachytherapy results in improved local control and decreased high-grade toxicities compared to historical controls. Incorporating MRI into the workflow of a department can be a major challenge when initiating an MRI-based brachytherapy program. This project aims to describe the goals, challenges, and solutions when initiating an MRI-based cervical cancer brachytherapy program at our institution.

Material and methods: We describe the 6-month multi-disciplinary planning phase to initiate an MRI-based brachytherapy program. We describe the specific challenges that were encountered prior to treating our first patient.

Results: We describe the solutions that were realized and executed to solve the challenges that we faced to establish our MRI-based brachytherapy program. We emphasize detailed coordination of care, planning, and communication to make the workflow feasible. We detail the imaging and radiation physics solutions to safely deliver MRI-based brachytherapy. The focus of these efforts is always on the delivery of optimal, state of the art patient care and treatment delivery within the context of our available institutional resources.

Conclusions: Previous publications have supported a transition to MRI-based brachytherapy, and this can be safely and efficiently accomplished as described in this manuscript.

Key words: brachytherapy, cervical cancer, image-based brachytherapy, MRI.

\section{Purpose}

Cervical cancer is the fourth most prevalent malignancy in females worldwide with an estimated 520,000 new diagnoses and 270,000 deaths annually [1]. Standard management of locally advanced cervical cancer includes external beam radiotherapy (EBRT) with concurrent cisplatin-based chemotherapy and brachytherapy (BT) $[2,3,4,5,6,7,8]$. Classically, BT was prescribed using the Paris and Stockholm method of milligram Radium-hours or the Manchester method to point A using orthogonal radiographs for treatment planning $[9,10,11]$. A more modern approach uses 3-dimensional (3D) - based treatment planning, either with computed tomography (CT) or magnetic resonance imaging (MRI). 3D BT allows for dose-volume evaluation of the target and the organs at risk (OARs). MRI-based treatment planning has superior soft tissue contrast compared to $\mathrm{CT}$, which allows for more accurate delineation of the gross tumor volume (GTV) and the high-risk clinical target volume (HR-CTV)
$[12,13]$. Regardless of the planning technique chosen, BT is a critical component of curative intent treatment of cervical cancer, and cannot be replaced by EBRT even with newer techniques $[14,15,16,17]$.

MRI-based BT is time, labor, and resource intensive, and therefore, the workflow to deliver state-of-the-art MRI-based adaptive BT can be challenging. The aim of this project is to provide the challenges and solutions that our institution experienced when developing an MRIbased BT program with the goal of making MRI-based BT more accessible to other institutions.

\section{Material and methods \\ Clinical challenges}

The team and first steps

In January 2014, our institution began the transition from a CT-based to MRI-based cervical BT program. The goal

Address for correspondence: Matthew Harkenrider, MD, Assist. Prof., Department of Radiation Oncology, Received: 16.01 .2017 Stritch School of Medicine Loyola University Chicago, Cardinal Bernardin Cancer Center, 2160 S $1^{\text {st }}$ Ave, Accepted: 28.02 .2017 Maguire Center, Room 2944, Maywood, IL 60153, Chicago, USA, phone: +1 708 216-2575, Published: 28.04 .2017 
of the program was to deliver state-of-the-art BT for our cervical cancer patients, on par with the treatment delivered in the pioneering centers around the world. Many potential challenges were realized when initiating the process to establish the MRI-based BT program. It would truly take a team approach with support from multiple departments (radiation oncology, gynecologic oncology, radiology, anesthesia) for the endeavor to be viable and successful.

\section{Education}

Historically, our radiation oncologists (MH/WS) treated cervical cancer patients with CT-based BT without first-hand experience in MRI-based BT. It was our institutional standard to perform 5 CT-based high-dose-rate (HDR) implants as an outpatient on non-consecutive days. For this reason, we needed to educate our collaborating physicians, physicists, and ourselves. We needed to determine the best applicator, dose-fractionation schedule, and prescription volume to develop an efficient workflow with optimal utilization of resources. Once we established the "big picture" aspects of initiating the MRI-based BT program, we turned attention to the details of the procedure. These would include materials (applicator, packing, contrast materials, etc.), MRI-related patient safety (applicator testing, contraindications to MRI, etc.), and inpatient management of BT patients (pain management, diet, bed rest, bowel management, etc.).

\section{Coordination and workflow}

Our center is spread out with an outpatient center at one end and the main hospital at the other end of the medical campus. Each location has an operating room and 1.5 or 3 Tesla (T) MRI units, but the HDR remote afterloader is in the department of radiation oncology located in the outpatient center. The hospital and the department of radiation oncology are not connected, which necessitates ambulance transport for patients being transferred between the two. The logistics regarding procedural location required collaboration and troubleshooting to determine the optimal workflow. We had to determine if the applicator placement, MRI acquisition, CT acquisition, and treatment delivery could be performed successfully without displacement of the applicator during patient transfers.

\section{Physics challenges}

\section{Applicator and magnetic resonance imaging compatibility}

The presence of a BT applicator when scanning with MRI raised multiple questions/concerns:

1. Is the applicator MRI safe, conditional, or unsafe?

2. If the applicator is MRI conditional, can those conditions be met given the specific MRI system and scan position of the applicator within the patient?

3. Will the applicator cause image artifacts and/or distortions? If yes, how can they be characterized and minimized?

Medical devices are classified into three categories with regards to MRI safety. Briefly, MRI safe devices pose no known hazards in all MRI environments. Magnetic resonance imaging unsafe devices are known to pose hazards in all MRI environments. Finally, MRI conditional devices have been demonstrated to pose no known hazards in a specified MRI environment with specified conditions of use. The bulk of medical devices fall into the MRI conditional category, which requires medical staff to interpret whether the device can be safely scanned given the MR imager and associated equipment, the location of the device within the patient, and the scanning region of the patient [18]. For MR conditional applicators, the manufacturer documentation should list a maximum safe (or list of approved) magnetic field strength(s) and a maximum safe static spatial gradient field to mitigate magnetically induced displacement force and torque risks. The documentation will also typically list a maximum specific absorption rate (SAR) to be used, which correlates to the amount of radiofrequency energy introduced into the patient. This information then needs to be compared to the corresponding sections in the MRI system(s) documentation to determine if and how the applicator can be scanned safely $[18,19]$.

It is also important to determine if the applicator will create artifacts or distortions in the resulting MR images. A device can be MRI conditional or even MRI safe and still cause image artifacts. In addition, the degree of artifact/ distortion in the images will vary depending on the position and orientation of the applicator with respect to the MRI scanner, the specific MRI pulse sequence used, and the parameters selected for that pulse sequence (repetition time, echo time, bandwidth, resolution, etc.). Ideally, testing the applicator within an imaging phantom is best for determining the severity of artifacts and distortions that can be expected in vivo. The applicator we used was the titanium MR conditional Fletcher-Suit-Delclos tandem and ovoid set (flexible geometry), model AL13030001 (Varian Medical Systems, Palo Alto, CA, USA). Upon the provision of the Vienna-style applicator in May 2016, we incorporated use of the Vienna-style 3D Interstitial Ring Applicator Set 60, model GM11010190 (Varian Medical Systems, Palo Alto, CA, USA).

\section{Magnetic resonance imaging safety}

Patient and staff safety must be considered during the MRI procedure. Most radiation oncology staff are unfamiliar with MRI safety precautions, and most radiological staff are unfamiliar with radiation therapy planning, especially in patients with an implanted applicator. Education, training, and adherence to a procedure proto$\mathrm{col}$ are necessary to ensure patient and staff safety while imaging the patient for treatment planning.

\section{Sequence acquisition}

Magnetic resonance imaging is often employed during the workup and staging and pre-BT assessment of cervical cancer patients. However, the imaging protocol must be modified for the purposes of BT planning, especially when the patient has an MRI conditional applicator, to stay within manufacturer-specified safety limits. The MR images must then be made available for contouring in the treatment planning software and subsequent registration to the CT dataset. We also recognized that this applicator 
can cause artifacts in the resulting images [20,21]. These applicator-induced artifacts can include signal loss, geometric distortion, and bright signal pile-up, and are caused by significant magnetic susceptibility differences between the applicator and the surrounding tissue [22,23]. Given the limitations of MRI and the potential for applicator-induced artifacts [20,21], we would have to understand our imaging limitations with an applicator in place for accurate contouring and registration.

\section{Results}

\section{Clinical solutions}

\section{The team and first steps}

After the initial phases of planning in January 2014, we met with our colleagues in radiology, gynecologic oncology, and anesthesia to explain our goals and solicit support for all the hard work and planning that this program would entail. We are fortunate that support from our colleagues was immediate and wholehearted, which was the foundation for our ultimate success. Our team consisted of 2 radiation oncologists (MH, WS), 2 gynecologic oncologists (RP, ML), 2 radiologists (JY, AG), 1 MRI physicist (SS), 1 MRI radiation oncology physicist (AW), 4 clinical physicists (MS, BC, MM, JR), and additional support from our nurses, radiation therapists, and administrators within the hospital system. Regular communication within our group was maintained throughout the initiation process and remains vital even in our more established program.

\section{Education}

The authors began thoroughly reviewing the literature of the various aspects of MRI-based cervical BT. Additionally, our radiation oncologists have taught at and attended (WS and $\mathrm{MH}$, respectively) the American Brachytherapy Society MRI-based cervical BT contouring course [24]. The best practice was described in the "An International Study on MRI-guided Brachytherapy in Locally Advanced Cervical Cancer" (EMBRACE) trial [25]. We also wrote a prospective study, so we could track and report our outcomes (LU 206907). Ultimately, our interest in image-based BT led us to write a review article emphasizing MRI-based BT for the treatment of cervical cancer [26].

We first determined that in order to make the best use of our operative and imaging resources, we would try to minimize the number of applicator placements and MRIs. We decided that a 4 -fraction course in 2 implants separated by 1-2 weeks would be optimal. This schedule has been well described by the University of Vienna group with excellent results $[27,28]$. It is also more convenient for patients who must travel for their BT, and it was our hope to make BT more accessible to the community that we serve. The drawback from this schedule is that it requires multiple patient transfers over the course of 2 days, as the patient travels from the hospital to the radiation oncology department and back each day. The multiple transfers of the patient can increase inter-fraction motion of the applicator and uncertainty of dose delivery [29]. There are many uncertainties regarding MRI-based cervical brachytherapy, and it is important to understand them and how to minimize them [30]. MRI-based BT requires increased physician attention as delineation of HR-CTV and OARs is needed. The minimum dose to $90 \%$ of the HR-CTV ( $D_{90}$ HR-CTV) is the most commonly prescribed target, since it is less sensitive to small variations in contouring [31]. Our institutional standard target dose in the CT era was $80-85$ Gy in 2 Gy equivalent fractions $\left(\mathrm{EQD}_{2}\right)$ to point A. Our MRI-based BT initial dose to $\mathrm{D}_{90} \mathrm{HR}$-CTV was also 80-85 Gy EQD 2 , but increased to $\geq 87$ Gy based on data reported by Dimopoulos et al. as we gained experience [32]. We have since evolved to target $\mathrm{D}_{90} \mathrm{HR}-\mathrm{CTV}$ of 90-95 Gy, as we utilize EMBRACE 2 planning goals with incorporation of a Vienna-style interstitial tandem and ring applicator [33]. Delineation of target volumes is beyond the scope of this report, but for more information the authors would refer readers to the recommendations from the The Groupe Européen de Curiethérapie and the European Society for Radiotherapy \& Oncology (GEC-ESTRO) [12,31].

\section{Coordination and workflow}

There are many steps in the process that we utilized at our institution. On Day 1, we place the applicator in the operating room, obtain the MRI, and perform treatment planning and delivery. On Day 2, we deliver the second fraction, remove the applicator, and subsequently discharge the patient. Our detailed workflow, including time estimates for steps longer than 5 minutes is shown in Figure 1. To make treatment days easier, we found it most efficient to establish a checklist for everything that required coordination prior to the procedure, which is shown in Figure 2.

We decided to perform the procedure and MRI in the main hospital on Day 1. The MRI checklist is obtained preoperatively. Our process begins with induction of general anesthesia and exam under anesthesia. We dilate the cervical os and place the tandem using transabdominal ultrasound guidance to minimize the chance of uterine perforation [34,35]. Two gold seeds are placed at approximately 3 and 9 o'clock positions in the cervix. The vaginal applicator (ovoids or ring) is placed, and the system is assembled and packed by the radiation oncologist. After packing is complete, the patient is returned to the supine position and orthogonal radiographs are taken to ensure proper applicator positioning [36]. We tried several iterations of our packing strategy with gauze soaked in different dilution ratios of gadolinium, dry gauze, and ultrasound gel-soaked gauze. We settled on using ultrasound gel-soaked gauze, as this has been shown to improve the contrast ratio [37]. GEC-ESTRO recommends using dry packing though for $\geq 1.5 \mathrm{~T}$ MRI and diluted gadolinium for $0.2 \mathrm{~T}$ MRI [38].

Following verification of proper applicator placement, the patient is awakened and recovered from general anesthesia. Recovery generally takes about 1 hour. Intravenous narcotic pain medication is used in recovery. During the hospital admission, the patient is transitioned to oral narcotic pain management as needed. Adequate 
Day 1

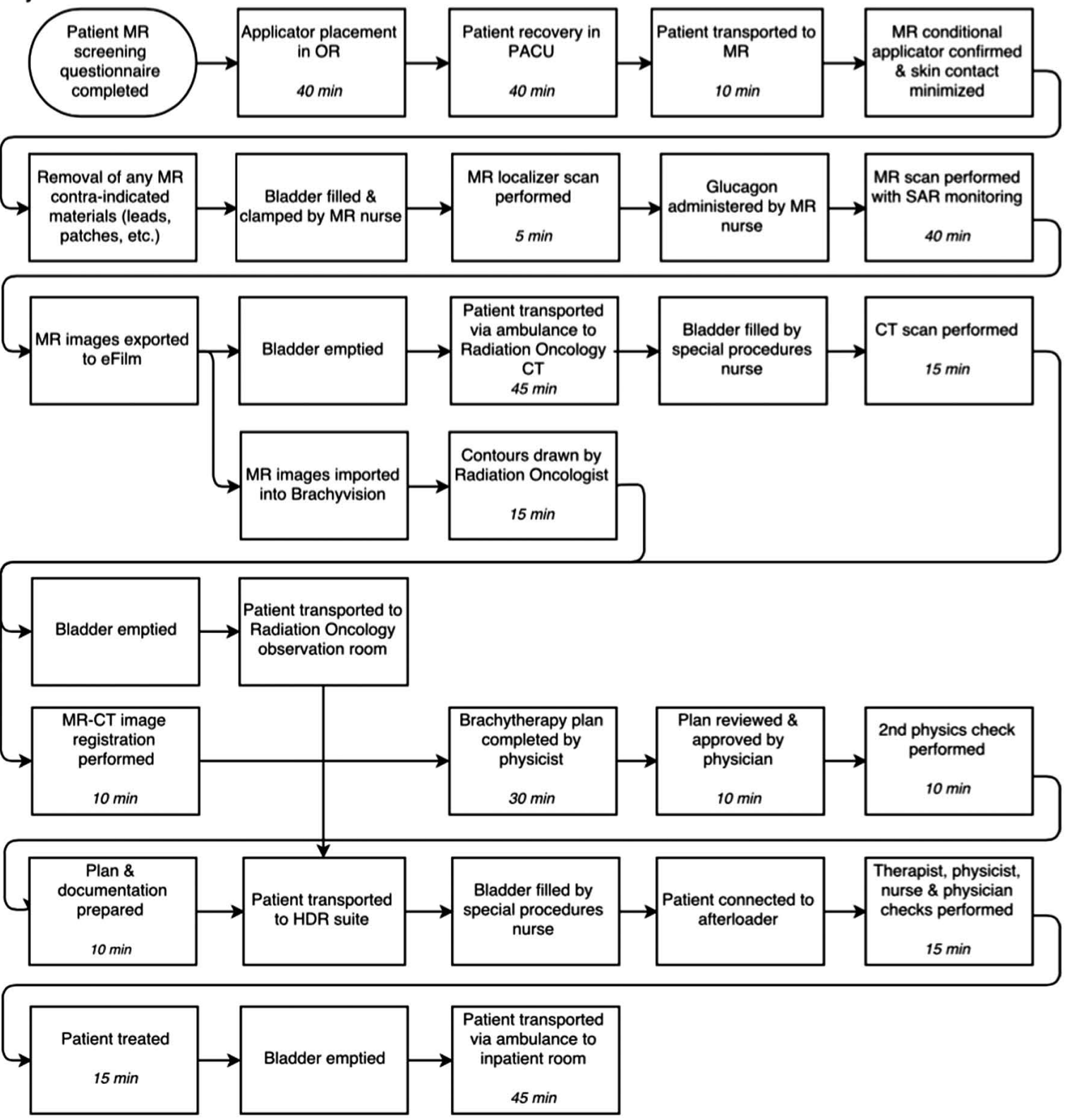

Day 2

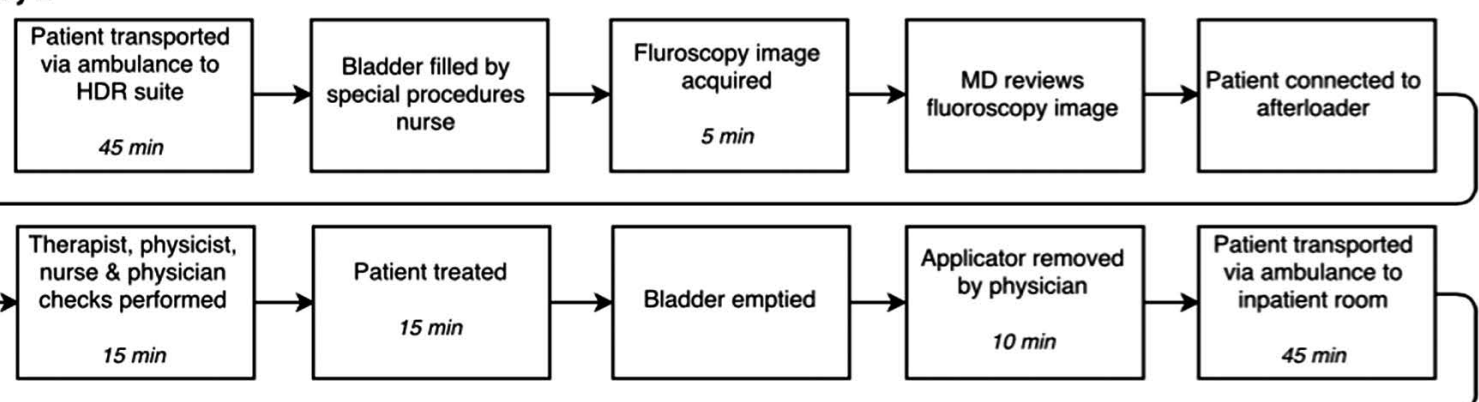

Patient discharged

Fig. 1. Procedural workflow for cervical brachytherapy studies. All steps longer than 5 minutes are indicated 
pain control is important so that the patient is both comfortable, and so she can hold still during imaging. Some centers have favored epidural anesthesia with good pain control $[39,40]$. Epidural anesthesia may be associated with undesirable effects including perioperative morbidity [41] and emotional stress related to time spent immobilized in the supine position with the applicator in place between treatment fractions [42].

Following applicator placement and recovery, the patient proceeds to MRI immediately. Magnetic resonance imaging availability can be a significant hurdle, but we provide notice to our MRI staff several weeks in advance to ensure MRI availability the morning of the procedure. In addition, we are scanning patients on an MR system that routinely handles inpatients, and therefore has builtin schedule flexibility to accommodate inpatient exams. We implemented a system using an MRI compatible padded transfer board to minimize motion due to patient transfers from MRI to CT. The padded transfer board is set up on the MR imaging table, and the patient is transferred onto the board prior to MR imaging. Details of the MRI safety and imaging protocols are discussed later. Glucagon $1 \mathrm{mg}$ IV is administered immediately prior to image acquisition to minimize internal motion from bowel motility. The patient then remains on this padded transfer board during transportation to the department of radiation oncology for $\mathrm{CT}$ scanning. We found that using this technique minimized the motion between acquisition of MR and CT images, allowing for more reliable registration.

Once the CT data is imported into the treatment planning software, digitally reconstructed radiographs (DRRs) were generated from this data. CT data is used for reconstruction of the applicator, while MRI data is used for contouring the target volumes and OARs. Discussions with the radiologists were commonly used to assist with target volume delineation for the first several patients. Treatment planning then commences with initial planning to deliver 7 Gy to point $A$. The dose is then optimized to increase the minimum dose to $\mathrm{D}_{90}$ HR-CTV to the goal of $\geq 87$ Gy initially, and more recently $90-95$ Gy, EQD 2 for the entire course of treatment $[33,43,44]$. Once treatment planning is complete, the treatment is delivered, and the patient is transferred to the hospital. She stays overnight and is managed with narcotic pain medications, anti-emetics, and loperamide. We do not have routine dietary restrictions during admission.

On Day 2, the patient is again transferred to the department of radiation oncology for delivery of the second fraction. KV images are acquired and compared to intraoperative radiographs to verify the applicator position. The gold seeds implanted at the time of applicator placement can be used to verify stable positioning as well. In order to limit variation in bladder volume, we use a consistent bladder filling protocol of $30 \mathrm{ml}$ prior to each imaging modality and prior to treatment. We do not acquire a CT prior to treatment on Day 2. We acknowledge that there may be internal variation of the sigmoid and rectum, but there can be variation of these organs in the time between the scan and first treatment and even during treatment. This is an uncertainty that we understand and chose to accept [29].
MRI-Based Brachytherapy Checklist

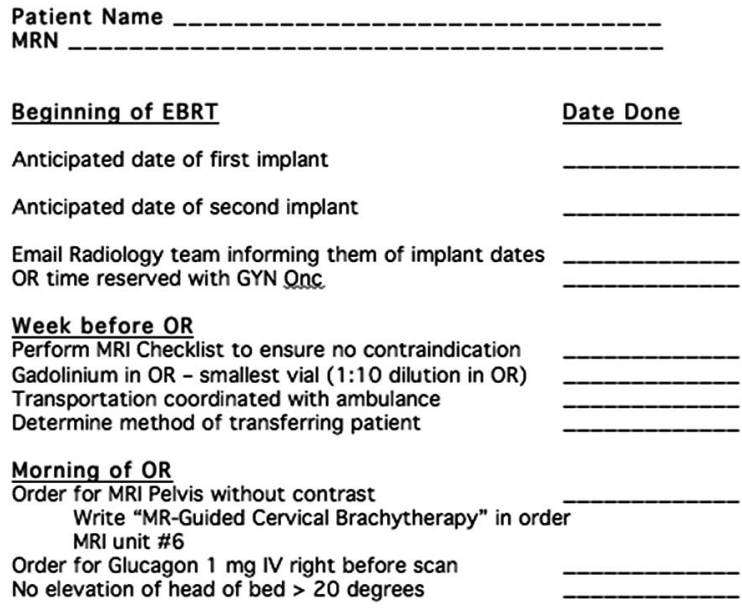

Fig. 2. Magnetic resonance imaging-based cervical brachytherapy pre-implant checklist

In July 2014, we treated our first MRI-based patient, and a radiation oncologist $(\mathrm{MH})$ and nurse practitioner followed the patient through the whole process. Subsequent patients were followed by our nurse practitioner throughout the process, and after about 10 patients, the process was seamless enough that each department operated independently to guide the patient through. Our program has evolved, and currently we have a special procedures nurse to supervise the patients' BT course. It was our ultimate goal to perform MRI-only planning (without CT), which has since been implemented. This strategy allows the patient to go to her hospital room after MRI, while her treatment planning is completed.

\section{Physics solutions}

\section{Applicator and MRI compatibility}

Since we chose an MRI conditional applicator, we referenced the manufacturer insert, which described the conditions under which the applicator was safe in the MR environment; these are summarized in Table 1 . We then had to assess, which MRI system at our institution, $1.5 \mathrm{~T}$ or $3 \mathrm{~T}$, was best suited for patient safety and image quality. The $3 \mathrm{~T}$ systems were ruled out for the following reasons:

1. Significantly lower maximum theoretically estimated whole body specific absorption rate (SAR) level allowed when using the applicator: $0.5 \mathrm{~W} / \mathrm{kg}$ at $3 \mathrm{~T}$ vs. $1.6 \mathrm{~W} / \mathrm{kg}$ at $1.5 \mathrm{~T}$.

2. SAR increases by a factor of 4 at $3 \mathrm{~T}$ compared to $1.5 \mathrm{~T}$, which means that imaging protocols must be run at lower flip angles or larger TR's (recovery time) at $3 \mathrm{~T}$ in order to match SAR levels at $1.5 \mathrm{~T}$. This negatively impacts signal-to-noise and image acquisition time.

3. Susceptibility artifacts increase with field strength; thus, these artifacts are worse at $3 \mathrm{~T}$ vs. $1.5 \mathrm{~T}$.

Magnetic resonance imaging was performed on a Magnetom Aera 1.5T MRI (Siemens Healthcare, Erlangen, Ger- 
Table 1. Magnetic resonance imaging conditional criteria for the Varian model AL13030001 applicator set

\begin{tabular}{|c|c|}
\hline Maximum static magnetic field strength & $3 T$ \\
\hline $\begin{array}{l}\text { Maximum spatial gradient of the static magnetic } \\
\text { field }\end{array}$ & $154 \mathrm{~T} / \mathrm{m}$ \\
\hline $\begin{array}{l}\text { Maximum product of static magnetic field \& spatial } \\
\text { gradient }\end{array}$ & $418 \mathrm{~T}^{2} / \mathrm{m}$ \\
\hline Maximum local body SAR, $1.5 \mathrm{~T}$ & $2.0 \mathrm{~W} / \mathrm{kg}$ \\
\hline $\begin{array}{l}\text { Maximum theoretically estimated whole body } \\
\text { SAR, } 1.5 \mathrm{~T}\end{array}$ & $1.6 \mathrm{~W} / \mathrm{kg}$ \\
\hline Maximum local body SAR, $3 \mathrm{~T}$ & $0.7 \mathrm{~W} / \mathrm{kg}$ \\
\hline $\begin{array}{l}\text { Maximum theoretically estimated whole body } \\
\text { SAR, } 3 \text { T }\end{array}$ & $0.5 \mathrm{~W} / \mathrm{kg}$ \\
\hline
\end{tabular}

MRI-magnetic resonance imaging, $T$ - Tesla, $m$ - meter, $W$ - Watt, $\mathrm{kg}$ - kilogram SAR - specific absorption ratio

many). Maximum values related to the static magnetic field strength of the MRI (Table 2) were safely below the maximum allowed values for the applicator. All MRI systems have software controls in place to adhere to the United States Food and Drug Administration and the International Electrotechnical Commission's guidelines for SAR $[45,46]$. However, in the lowest software setting, normal mode, the whole body SAR will only be limited to an average of $2 \mathrm{~W} / \mathrm{kg}$. This value is higher than the maximum theoretically estimated whole body SAR recommended by the manufacturer for the applicator at $1.5 \mathrm{~T}$. This means that the estimated whole body SAR must be manually checked by the technologist before each pulse sequence, in order to stay within safety guidelines. This is described in more detail in the MRI safety section.

Phantom imaging can assess the magnitude of artifacts of the titanium applicators in MRI. We developed a phantom holder (Figure 3) similar to those previously described

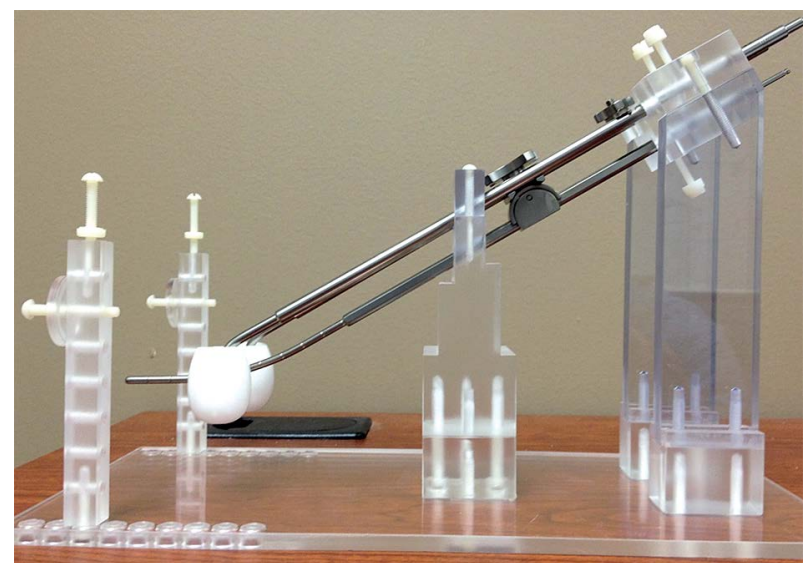

Fig. 3. Sagittal view of the phantom holder with the T\&O applicator inserted. The holder was then placed in a plastic tub (Velcro was used to adhere the holder bottom to the tub). The tub was filled with a $0.1 \mathrm{mM}$ solution of Gadobutrol in distilled water to obtain an approximate T1 of 1.2 seconds at $1.5 \mathrm{~T}$
Table 2. Relevant static magnetic field parameters for the Siemens Magnetom Aera 1.5 T MRI scanner Operating static magnetic field strength: $1.5 \mathrm{~T}$

\begin{tabular}{l}
$\begin{array}{l}\text { Maximum spatial gradient of the static } \\
\text { magnetic field }\end{array}$ \\
\hline $\begin{array}{l}\text { Maximum product of static magnetic field } \\
\text { \& spatial gradient }\end{array}$ \\
$\begin{array}{l}T \text { - Tesla, MRI - magnetic resonance imaging, } m \text { - meter } \\
\text { ( } \mathrm{m}( \pm 10 \%)\end{array}$
\end{tabular}

$[47,48]$ and imaged the applicator on MRI and CT to evaluate artifacts and distortions. The tandem and ovoid applicator was segmented on axial CT images, which were then registered to the MR images in the treatment planning software. The applicator volume was then overlaid on all MRI sets in the open source software, 3D-slicer [49], and distances were measured from the tandem tip to the MRI artifact edge in right/left/superior and anterior/ posterior/superior directions from coronal and sagittal acquisitions. Artifact regions were also manually contoured in coronal/sagittal orientations for area measurements. As would be expected, reductions in voxel size and increases in readout bandwidth reduced artifact size (average max artifact length decreased by $0.95 \mathrm{~mm}$ and average max area decrease by $\left.0.27 \mathrm{~cm}^{2}\right)$. Interestingly, bandwidth increases yielded reductions in area $\left(0.19 \mathrm{~cm}^{2}\right)$ and in distance measurements $(1 \mathrm{~mm})$ even with increases in voxel size, as compared to a standard imaging protocol [50].

\section{Magnetic resonance imaging safety}

Since the applicator is MRI conditional, it is imperative that the correct MRI scanner and imaging protocol are used for these patients. The steps below detail the MRI scanning procedure that is followed for scans with the MR conditional applicator inserted into a patient. A checklist was also created for use during studies to ensure the correct safety procedure is followed as shown in Figure 4. The stored imaging protocol was designed to minimize SAR, while maintaining adequate image quality. Each pulse sequence was configured to include a pause after preparation, so that the technologist can check the predicted SAR values. For each acquisition, SAR is targeted to be at or below $1.6 \mathrm{~W} / \mathrm{kg}$ to meet the MRI conditional guidelines of the applicator. Before starting each MR image acquisition, the technologist checks the predicted whole body SAR value using the MR software to ensure it is less than $1.6 \mathrm{~W} / \mathrm{kg}$. If it is greater, the technologist modifies the MRI pulse sequence parameters to reduce the predicted SAR and repeat the procedure above. If the parameters cannot be modified to reduce the predicted SAR to $1.6 \mathrm{~W} / \mathrm{kg}$ or less, that image acquisition is deferred and the technologist proceeds to the next MRI pulse sequence; however, we have never encountered this situation in our patients. After the final image acquisition and before the patient was removed from the scanner, the technologist records the SAR operating mode as a final safety check. The MR physicist (SS) reviewed the recorded SAR value at the time of scanning for the first three patients to ensure that SAR conditions 


\section{Appendix A: Checklist for MRI of Patients with Varian MR Conditional Titanium Fletcher-Suit-Delclos-style Applicator Set Internal Radiology Record}

\section{Staff Preparation}

$\square$ Confirm External Staff have been screened using MR staff safety questionnaire

$\square$ External Staff removes all MR contra-indicated materials (pagers, cell phones, wallets, pens, ID's, etc.) if they will enter Zone IV

\section{Patient Preparation}

$\square$ Confirm with RadOnc staff and with visual inspection (look for Ti label) that Varian MRconditional Titanium Fletcher-Suit-Delclos-style applicator (AL13030001) is present

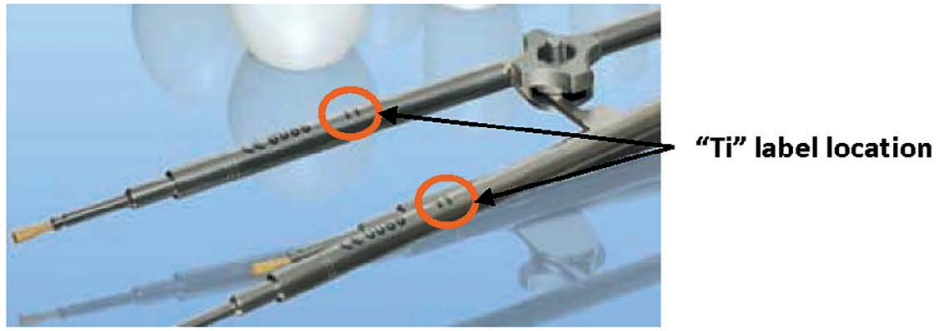

$\square$ Document applicator used on MR-patient screening form (Varian MR-conditional T\&O applicator, AL13030001)

$\square$ RadOnc staff confirm all extraneous metal parts (obdurators/mandrins, marker clips, marker wires, length gauges, etc.) have been removed

$\square$ Applicator is not in direct contact with patient skin

$\square$ Patient is checked for MR contra-indicated materials

\section{MR Imaging}

$\square$ SAR monitor is set to normal mode

$\square$ Every protocol is set to include pause before imaging

$\square$ Every protocol is manually checked for SAR to be below $0.72 \mathrm{~W} / \mathrm{lb}(1.6 \mathrm{~W} / \mathrm{kg})$

$\square$ Record SAR operating mode before removing the patient:

Patient Name:

MR Technologist Name:

MR Technologist Signature:
MRN:

Date:

Fig. 4. Magnetic resonance imaging procedure safety checklist

were followed. For all subsequent studies, random reviews of patient images were performed to ensure that the SAR limit is not exceeded. For reviews of 38 different MRI studies across 27 patients, the SAR limit was not exceeded for any pulse sequence run.

Magnetic resonance image acquisition and planning

Magnetic resonance images are acquired with two main goals: delineating the target and minimizing artifacts from the titanium applicator. Standard practice with
MRI-based BT involves using 2D T2-weighted fast spin echo pulse sequences to visualize the gross target volume, which tends to appear hyper-intense relative to normal tissue. Artifacts produced by the applicator can not only obscure the diseased portions of the anatomy, but can also yield inaccuracies in catheter reconstruction if using MRI-only planning. We attempted to minimize this by using fast spin echo sequences and optimizing pulse sequence parameters in phantom studies. In addition, we started this program with the intent of doing MRI- 
Table 3. Magnetic resonance imaging protocol: MRI protocol for cervical brachytherapy planning. Version 1 is the original protocol that at the initiation of our MRI-based cervical brachytherapy program. Version 2 is the subsequent optimized version that was implemented after testing in patients [50]. The changes in readout bandwidth and spatial resolution reduced applicator-induced artifact with minimal signal-to-noise loss and without increasing imaging time

\begin{tabular}{|c|c|c|c|c|c|c|c|}
\hline Name & TR (ms) & TE (ms) & ETL & Flip angle & Avgs & $\begin{array}{l}\text { Readout BW } \\
(\mathrm{Hz} / \mathrm{px})\end{array}$ & $\operatorname{Res}\left(\mathrm{mm}^{3}\right)$ \\
\hline \multicolumn{8}{|l|}{ Version 1} \\
\hline $\begin{array}{l}\text { 2D spoiled gradient echo localizer: } \\
\text { sag/cor/axial }\end{array}$ & 7.8 & 2.94 & NA & 20 & 1 & 450 & $1.90 \times 1.33 \times 8.0$ \\
\hline 2D T2 FSE cervix: sag/cor/axial & 3000 & 91 & 15 & 120 & 1 & 200 & $0.98 \times 0.78 \times 4.0$ \\
\hline $\begin{array}{l}\text { 3D T2 variable flip angle FSE cervix: } \\
\text { axial }\end{array}$ & 1700 & 96 & 80 & 170 & 2 & 630 & $0.98 \times 0.98 \times 1.4$ \\
\hline \multicolumn{8}{|l|}{ Version 2} \\
\hline $\begin{array}{l}\text { 2D spoiled gradient echo localizer: } \\
\text { sag/cor/axial }\end{array}$ & 7.8 & 2.94 & NA & 20 & 1 & 450 & $1.90 \times 1.33 \times 8.0$ \\
\hline 2D T2 FSE cervix: sag/cor/axial & 3600 & 91 & 25 & 120 & 1 & 501 & $0.98 \times 0.98 \times 4.0$ \\
\hline $\begin{array}{l}\text { 3D T2 variable flip angle FSE cervix: } \\
\text { axial }\end{array}$ & 1700 & 96 & 80 & 170 & 2 & 630 & $0.98 \times 0.98 \times 1.4$ \\
\hline
\end{tabular}

$T R$ - recovery time, TE - echo time, ETL - echo train length, Avgs - averages, BW-bandwidth in Hertz (Hz) per pixel (px), Res - resolution, $m m^{3}$ - cubic millimeters, $2 D$ - two-dimensional, sag - sagittal, cor-coronal, FSE - fast spin echo, 3D - three-dimensional

based planning with CT for applicator reconstruction as opposed to an MRI-only planning. Therefore, we also ran a 3D MRI sequence to aid in the co-registration of CT images to MR images. Thus, the MR images are used to identify the targets and OAR, and the CT data was used for applicator reconstruction and treatment planning. Detailed information on our imaging protocol can be found in Table 3.

Magnetic resonance imaging and CT images are exported to the planning software, where physicians then contour target volumes and OAR. T2-weighted images are acquired in an oblique axial orientation (perpendicular to the tandem and in the plane of the cervix) and are used for contouring. Obtaining MR images in this orientation was part of the instructions for the MR image protocol, and it is the standard acquisition orientation for diagnostic scans of the cervix. We use image registration software within the treatment planning system (Eclipse/ BrachyVision, Varian Medical Systems, Palo Alto, CA, USA) to co-register the CT to the MR image data. Initially, we were concerned that there would be some discrepancies between the MR and CT data due to the curved MR tabletop, but in our experience this has not been an issue. Rigid registration is sufficient for our purposes since the padded transfer board minimizes patient motion between transfers, and presumably limits the degree of deformation due to the curvature of the MR tabletop. Image registration is performed using the axial oblique T2-weighted sequences as the source image and CT data as the target image. Data from the other MR pulse sequences is used to fine-tune the registration, especially the 3D with isotropic voxels, since these can be reformatted to match the imaging plane of the CT data. Applicator-induced artifacts and the subsequent impact on the delineation of applica- tor and source positions has not been an issue, as we initially reconstructed the applicator using the CT. The artifact has not seemed to significantly affect the quality of our image registration. Prior to transitioning to MRI-only planning, 3 of our radiation therapy physicists (AW, BC, $\mathrm{MM})$ re-planned the first 10 patients, blinded to the CT scans, using only the MRI data sets. We compared the resulting plans with the patients' MRI/CT-based plans. We found no clinically meaningful differences in doses to the HR-CTV or OAR with addition of MRI/CT-based plans, so we have since performed MRI-only planning [51].

\section{Discussion}

Magnetic resonance imaging-based cervical BT is a labor and resource intensive treatment modality. It requires significant coordination of care and effective communication to be thorough and efficient. The treatment delivery is very rewarding, since the treatment plan is of the highest possible quality and individualization for the patient. Each institution has its own challenges when starting an MRI-based BT program, which can be overcome with hard work and a dedicated multi-disciplinary team.

We described the processes that worked best for our institution. However, there are various methods that may be employed when performing MRI-based HDR intracavitary BT for the treatment of cervical cancer. Gill et al. described the utilization of MRI for the first fraction after applicator insertion with CT used for the remaining fractions [52]. Alternatively, Potter et al. demonstrated a methodology, in which a pre-treatment MRI is performed prior to performing an implant. Their study found that the pre-BT MRI helped define HR-CTV, especially compared to CT images with the applicator in place [53]. Tait 
et al. utilized a similar approach with a pre-implantation MRI, which was fused to the planning CT. The GTV and HR-CTV were contoured on the MRI, and a separate HR-CTV was made from the deformable image registration of the pre-implantation MRI to the planning CT [54]. Trifiletti and colleagues discussed yet another method, in which patients have a Smit sleeve placed after their first BT fraction, and an MRI is obtained with the Smit sleeve, but without the applicator in place. For subsequent fractions, CT is performed with the applicator in place, and these CT scans are fused to the MRI using the Smit sleeve for rigid body registration [55]. Other groups have published methods to facilitate implementation of MRI into a cervical BT program [56,57].

Regardless of what method is used to incorporate MRI into a cervical BT program, such advanced imaging and treatment planning allows for optimal individualization of BT. Our program has continued to evolve with incorporation of a Vienna-style interstitial tandem and ring allowing for even tighter constraints to OAR and while delivering an increased $D_{90}$ HR-CTV. We hope that our experience can help other institutions establish their own MRI-based BT programs.

\section{Acknowledgements}

James Ryva and Colleen Schaidle were instrumental in establishing this program.

\section{Disclosure}

Authors report no conflict of interest.

\section{References}

1. GLOBOCAN Cervical Cancer Fact Sheet. Available at http:// globocan.iarc.fr/old/FactSheets/cancers/cervix-new.asp Accessed: 12 January 2017.

2. Morris M, Eifel PJ, Lu J et al. Pelvic radiation with concurrent chemotherapy compared with pelvic and para-aortic radiation for high-risk cervical cancer. N Engl J Med 1999; 340: 1137-1143.

3. Rose PG, Bundy BN, Watkins EB et al. Concurrent cisplatin-based radiotherapy and chemotherapy for locally advanced cervical cancer. N Engl J Med 1999; 340: 1144-1153.

4. Whitney CW, Sause W, Bundy BN et al. Randomized comparison of fluorouracil plus cisplatin versus hydroxyurea as an adjunct to radiation therapy in stage IIB-IVA carcinoma of the cervix with negative para-aortic lymph nodes: A Gynecologic Oncology Group and Southwest Oncology Group study. J Clin Oncol 1999; 17: 1339-1348.

5. Eifel PJ, Winter K, Morris M et al. Pelvic irradiation with concurrent chemotherapy versus pelvic and para-aortic irradiation for high-risk cervical cancer: an update of radiation therapy oncology group trial (RTOG) 90-01. J Clin Oncol 2004; 22: 872-880.

6. Rose PG, Ali S, Watkins E et al. Long-term follow-up of a randomized trial comparing concurrent single agent cisplatin, cisplatin-based combination chemotherapy, or hydroxyurea during pelvic irradiation for locally advanced cervical cancer: A Gynecologic Oncology Group Study. J Clin Oncol 2007; 25: 2804-2810.

7. Keys HM, Bundy BN, Stehman FB et al. Cisplatin, radiation, and adjuvant hysterectomy compared with radiation and adjuvant hysterectomy for bulky stage IB cervical carcinoma. N Engl J Med 1999; 340: 1154-1161.
8. Stehman FB, Ali S, Keys HM et al. Radiation therapy with or without weekly cisplatin for bulky stage $1 \mathrm{~B}$ cervical carcinoma: follow-up of a Gynecologic Oncology Group trial. Am J Obstet Gynecol 2007; 197: 503 e501-506.

9. Walstam $\mathrm{R}$. The dosage distribution in the pelvis in radium treatment of carcinoma of the cervix. Acta Radiol 1954; 42: 237-250.

10. Pierquin B. Precis de curietherapie, endocurietherapie et plesiocurietherapie. Masson, Paris 1964.

11. Tod M, Meredith W. A dosage system for use in the treatment of cancer of the uterine cervix. Br J Radiol 1938; 1: 809-824.

12. Haie-Meder C, Pötter R, Van Limbergen E et al. Recommendations from Gynaecological (GYN) GEC-ESTRO Working Group (I): concepts and terms in 3D image based 3D treatment planning in cervix cancer brachytherapy with emphasis on MRI assessment of GTV and CTV. Radiother Oncol 2005; 74: 235-245.

13. Viswanathan AN, Dimopoulos J, Kirisits C et al. Computed tomography versus magnetic resonance imaging-based contouring in cervical cancer brachytherapy: results of a prospective trial and preliminary guidelines for standardized contours. Int J Radiat Oncol Biol Phys 2007; 68: 491-498.

14. Perez CA, Breaux S, Madoc-Jones H et al. Radiation therapy alone in the treatment of carcinoma of uterine cervix. I. Analysis of tumor recurrence. Cancer 1983; 51: 1393-1402.

15. Eifel PJ, Thoms WW, Jr., Smith TL et al. The relationship between brachytherapy dose and outcome in patients with bulky endocervical tumors treated with radiation alone. Int J Radiat Oncol Biol Phys 1994; 28: 113-118.

16. Viswanathan AN, Cormack R, Rawal B et al. Increasing brachytherapy dose predicts survival for interstitial and tandembased radiation for stage IIIB cervical cancer. Int J Gynecol Cancer 2009; 19: 1402-1406.

17. Tanderup K, Eifel PJ, Yashar CM et al. Curative radiation therapy for locally advanced cervical cancer: brachytherapy is NOT optional. Int J Radiat Oncol Biol Phys 2014; 88: 537-539.

18. Shellock FG, Woods TO Crues JV, 3rd. MRI labeling information for implants and devices: explanation of terminology. Radiology 2009; 253: 26-30.

19. Expert Panel on MR Safety, Kanal E, Barkovich AJ, Bell C et al. ACR guidance document on MR safe practices: 2013. J Magn Reson Imaging 2013; 37: 501-530.

20. Hellebust TP, Kirisits C, Berger D et al. Recommendations from Gynaecological (GYN) GEC-ESTRO Working Group: considerations and pitfalls in commissioning and applicator reconstruction in 3D image-based treatment planning of cervix cancer brachytherapy. Radiother Oncol 2010; 96: 153-160.

21. Soliman AS, Owrangi A, Ravi A et al. Metal artefacts in MRI-guided brachytherapy of cervical cancer. J Contemp Brachytherapy 2016; 8: 363-369.

22. Hargreaves BA, Worters PW, Pauly KB et al. Metal-induced artifacts in MRI. AJR Am J Roentgenol 2011; 197: 547-555.

23. Schenck JF. The role of magnetic susceptibility in magnetic resonance imaging: MRI magnetic compatibility of the first and second kinds. Med Phys 1996; 23: 815-850.

24. American Brachytherapy Society Meetings and Events. Available at: https:/ / www.americanbrachytherapy.org/meetings/ index.cfm. Accessed: 24 January 2016.

25. EMBRACE Download PDF Protocol. Available at: https:// www.embracestudy.dk/AboutProtocolDownload.aspx. Accesed: 6 December 2015.

26. Harkenrider MM, Alite F, Silva SR et al. Image-Based Brachytherapy for the Treatment of Cervical Cancer. Int J Radiat Oncol Biol Phys 2015; 92: 921-934.

27. Pötter R, Dimopoulos J, Georg P et al. Clinical impact of MRI assisted dose volume adaptation and dose escalation in brachytherapy of locally advanced cervix cancer. Radiother Oncol 2007; 83: 148-155. 
28. Pötter R, Georg P, Dimopoulos JC et al. Clinical outcome of protocol based image (MRI) guided adaptive brachytherapy combined with 3D conformal radiotherapy with or without chemotherapy in patients with locally advanced cervical cancer. Radiother Oncol 2011; 100: 116-123.

29. Nesvacil N, Tanderup K, Hellebust TP et al. A multicentre comparison of the dosimetric impact of inter- and intra-fractional anatomical variations in fractionated cervix cancer brachytherapy. Radiother Oncol 2013; 107: 20-25.

30. Tanderup K, Nesvacil N, Potter R et al. Uncertainties in image guided adaptive cervix cancer brachytherapy: impact on planning and prescription. Radiother Oncol 2013; 107: 1-5.

31. Pötter R, Haie-Meder C, Van Limbergen E et al. Recommendations from gynaecological (GYN) GEC ESTRO working group (II): concepts and terms in 3D image-based treatment planning in cervix cancer brachytherapy-3D dose volume parameters and aspects of 3D image-based anatomy, radiation physics, radiobiology. Radiother Oncol 2006; 78: 67-77.

32. Fokdal L, Tanderup K, Hokland SB et al. Clinical feasibility of combined intracavitary/interstitial brachytherapy in locally advanced cervical cancer employing MRI with a tan$\mathrm{dem} /$ ring applicator in situ and virtual preplanning of the interstitial component. Radiother Oncol 2013; 107: 63-68.

33. EMBRACE. Available at: https://www.embracestudy.dk/ About.aspx. Accessed: 11 January 2015.

34. Davidson MT, Yuen J, D'Souza DP et al. Optimization of high-dose-rate cervix brachytherapy applicator placement: the benefits of intraoperative ultrasound guidance. Brachytherapy 2008; 7: 248-253.

35. Small W Jr., Strauss JB, Hwang CS et al. Should uterine tandem applicators ever be placed without ultrasound guidance? No: a brief report and review of the literature. Int J Gynecol Cancer 2011; 21: 941-944.

36. Viswanathan AN, Moughan J, Small W Jr et al. The quality of cervical cancer brachytherapy implantation and the impact on local recurrence and disease-free survival in radiation therapy oncology group prospective trials 0116 and 0128 . Int J Gynecol Cancer 2012; 22: 123-131.

37. Swanick CW, Castle KO, Rechner LA et al. Optimizing packing contrast for MRI-based intracavitary brachytherapy planning for cervical cancer. Brachytherapy 2015; 14: 385-389.

38. Dimopoulos JC, Petrow P, Tanderup K et al. Recommendations from Gynaecological (GYN) GEC-ESTRO Working Group (IV): Basic principles and parameters for MR imaging within the frame of image based adaptive cervix cancer brachytherapy. Radiother Oncol 2012; 103: 113-122.

39. Janaki MG, Nirmala S, Kadam AR et al. Epidural analgesia during brachytherapy for cervical cancer patients. J Cancer Res Ther 2008; 4: 60-63.

40. Isoyama-Shirakawa Y, Nakamura K, Abe M et al. Caudal epidural anesthesia during intracavitary brachytherapy for cervical cancer. J Radiat Res 2015; 56: 583-587.

41. Lanciano R, Corn B, Martin E et al. Perioperative morbidity of intracavitary gynecologic brachytherapy. Int J Radiat Oncol Biol Phys 1994; 29: 969-974.

42. Kirchheiner K, Czajka-Pepl A, Ponocny-Seliger E et al. Posttraumatic stress disorder after high-dose-rate brachytherapy for cervical cancer with 2 fractions in 1 application under spinal/epidural anesthesia: incidence and risk factors. Int $\mathrm{J}$ Radiat Oncol Biol Phys 2014; 89: 260-267.

43. Dimopoulos JC, Potter R, Lang S et al. Dose-effect relationship for local control of cervical cancer by magnetic resonance image-guided brachytherapy. Radiother Oncol 2009; 93: 311-315.

44. Brachytherapy Guidelines. Available at http://www.american-brachytherapy.org/guidelines/index.cfm. Accessed:3December 2016.
45. US Food and Drug Administration. Criteria for Significant Risk Investigations of Magnetic Resonance Diagnostic Devices. June 20, 2014. Available at http://www.fda.gov/MedicalDevices/DeviceRegulationandGuidance/GuidanceDocuments/ucm072686.htm. Accessed: 14 December 2016.

46. IEC 60601-1-1, Medical electrical equipment - part 2: particular requirements for the safety of magnetic resonance equipment for medical diagnosis. International Electrotechnical Commission (IEC), 2010.

47. Haack S, Nielsen SK, Lindegaard JC et al. Applicator reconstruction in MRI 3D image-based dose planning of brachytherapy for cervical cancer. Radiother Oncol 2009; 91: 187-193.

48. Kim Y, Muruganandham M, Modrick JM et al. Evaluation of artifacts and distortions of titanium applicators on 3.0-Tesla MRI: feasibility of titanium applicators in MRI-guided brachytherapy for gynecological cancer. Int J Radiat Oncol Biol Phys 2011; 80: 947-955.

49. Fedorov A, Beichel R, Kalpathy-Cramer J et al. 3D Slicer as an image computing platform for the Quantitative Imaging Network. Magn Reson Imaging 2012; 30: 1323-1341.

50. Shea S, Diak A, Surucu M et al. Effect of Pulse Sequence Parameters on Geometric Distortions Induced by a Titanium Brachytherapy Applicator. Med Phys 2015; 42: 3313.

51. Chinsky B, Diak A, Small Jr W et al. Can Magnetic Resonance Imaging (MRI) Only Replace MRI-Computed Tomography Planning with a Titanium Applicator for Cervical Brachytherapy? Int J Radiat Oncol Biol Phys 2016; 96: S225-S226.

52. Gill BS, Kim H, Houser CJ et al. MRI-guided high-dose-rate intracavitary brachytherapy for treatment of cervical cancer: The University of Pittsburgh experience. Int J Radiat Oncol Biol Phys 2015; 91: 540-547.

53. Pötter R, Federico M, Sturdza A et al. Value of Magnetic Resonance Imaging Without or With Applicator in Place for Target Definition in Cervix Cancer Brachytherapy. Int J Radiat Oncol Biol Phys 2016; 94: 588-597.

54. Tait LM, Hoffman D, Benedict $S$ et al. The use of MRI deformable image registration for CT-based brachytherapy in locally advanced cervical cancer. Brachytherapy 2016; 15: 333-340.

55. Trifiletti DM, Libby B, Feuerlein $S$ et al. Implementing MRI-based target delineation for cervical cancer treatment within a rapid workflow environment for image-guided brachytherapy: A practical approach for centers without inroom MRI. Brachytherapy 2015; 14: 905-909.

56. Owrangi AM, Prisciandaro JI, Soliman A et al. Magnetic resonance imaging-guided brachytherapy for cervical cancer: initiating a program. J Contemp Brachytherapy 2015; 7: 417-422.

57. Koulis TA, Doll CM, Brown D et al. Implementation and validation of a combined MRI-CT-based cervical cancer brachytherapy program using existing infrastructure. Brachytherapy 2016; 15: $319-326$ 